\title{
Extending School-Wide Positive Behaviour Support (SWPBS) with Emotional Support Systems: A Non-Randomized Study Testing the Effectiveness of a School-Wide Health Care Policy TIME-IN in Special Education
}

\author{
Henk Weymeis ${ }^{1^{*}}$, Karla Van Leeuwen ${ }^{2} \&$ Caroline Braet $^{1}$ \\ ${ }^{1}$ Department of Developmental, Personality and Social Psychology, Ghent University, Belgium, \\ Henri Dunantlaan 2, 9000 Gent \\ ${ }^{2}$ Parenting and Special Education Research Unit, KU Leuven, Belgium, Leopold Vanderkelenstraat \\ 32 - bus 3765, 3000 Leuven
}

\begin{abstract}
Children's readiness for school is often threatened by the occurrence of both externalising (EP) and internalising (IP) problems. In search for solutions, research has shown that SchoolWide Positive Behaviour Support (SWPBS) is particularly effective for fostering children's behavioural skills and reducing EP. However, whether SWPBS can enhance children's emotional skills and reduce IP is less clear. Therefore, TIME-IN was developed, which extends SWPBS by also including emotional support systems. It will be tested whether TIME-IN is effective for (a) improving emotion regulation (ER) and (b) reducing depressive symptoms. Furthermore, it will be tentatively explored whether TIME-IN is accompanied by more than natural fluctuations in both children's EP and IP. The effectiveness of TIME-IN will be evaluated in a non-randomized study, in which an intervention group will be compared with a matched control group. Both research questions will be addressed in a sample consisting of children between 8 and 12 years of age with special educational needs (SEN), who have been argued to benefit most from school readiness interventions. Questionnaires for teachers, children, and their parents will be administered at the beginning (T0) and the end of the school year (T1) using multi-informant assessment. Practical implications, strengths, and limitations were discussed.
\end{abstract}

ISCRTN registry 54456609. Registered on 28 March 2017.

Keywords: school readiness, school-wide health care policy, emotion regulation strategies, internalising problems, externalising problems

Correspondence to Henk Weymeis, Henk.Weymeis@UGent.be, Department of Developmental, Personality and Social Psychology, Ghent University, Belgium Henri Dunantlaan 2, 9000 Ghent, Belgium

Special Research Fund Grant number: BOF.DC1.2016.0025.01.

Disclosure of competing interest: Braet, Cracco, \& Theuwis (2013) translated the FEEL-KJ (Grob \& Smolenski, 2005) and received an authorship fee for the official version. 


\section{Background}

To promote SEN children's school readiness (see Britto, 2012) and, more concretely, identify and reduce both EP and IP problems within the school environment, it seems appropriate to extend School-Wide Positive Behaviour Support (SWPBS) and its wellevaluated behavioural interventions (i.e. mainly intended for reducing EP; see e.g. Horner et al., 2009) with transdiagnostic mechanisms that explicitly focus on IP, such as emotion regulation (ER; Aldao, Nolen-Hoeksema, \& Schweizer, 2010; McIntosh, Ty, \& Miller, 2014). Emotion regulation (ER) was defined as "processes by which individuals influence which emotions they have, when they have them, and how they experience and express these emotions" (Gross, 1998; p. 224).

As a result, TIME-IN was developed, which complements SWPBS by adding emotional support systems such as (a) screening instruments for identifying IP, (b) ER training and (c) crisis intervention strategies. It was considered useful to train children's adaptive ER strategies by means of the key principles of Affect Regulation Training (ART; Berking \& Schwarz, 2014), since ART has several advantages as it complements behavioural and cognitive interventions (as present in SWPBS), integrates different adaptive ER strategies into one coherent model, and is effective for reducing various mental health problems (Berking \& Lukas, 2015). For the moment, ART has only been evaluated in adults, although it was claimed to be also applicable to younger age groups (Berking \& Schwarz, 2014). Furthermore, to support emotionally overwhelmed children, Life Space Crisis Intervention was used (LSCI; Long, Wood, \& Fecser, 2003), which was stated to fit well within a schoolwide approach (Dawson, 2003), and was found to be effective for children with SEN (Soenen, Volckaert, D'Oosterlinck, \& Broekaert, 2014). As such, TIME-IN is a school-wide health care policy, consisting of both behavioural and emotional support systems, with the final aim of promoting children's school readiness (Weymeis, 2015).

\subsection{The current study: evaluating TIME-IN}

TIME-IN will be implemented in a real-life setting (i.e. special education). In the current study, a practice-based evaluation will be performed to determine whether or not TIME-IN is effective for promoting children's school readiness (Veerman \& Van Yperen, 2007). More specifically, to be able to consider TIME-IN as 'potentially' effective, a clear description of its essential elements is required (e.g. goals, target group, methods, activities, and requirements). In order to meet this requirement, a full description of TIME-IN is available in a book (Weymeis, 2015), and will be shortly described in the methods section. Also, a CONSORT diagram with the numbers of participants recruited and tested in each condition will be provided (Simons et al., 2016). Next, we choose for a quasi-experimental design (i.e. non-randomized research) using a pre-post-test design, in which an intervention and a matched control group will be selected (Simons et al., 2016). According to Veerman and Van Yperen (2007), quasi-experimental research may yield signs of causality and, moreover, provide useful indications to judge whether or not an intervention is effective. However, at the same time, these indications should be interpreted with great caution, as possible alternative explanations for the observed results will not necessarily be ruled out.

The first goal of the current study will be to investigate whether TIME-IN is effective for improving child-reported emotional competencies and psychosocial well-being. More specifically, the confirmatory hypotheses will be tested that, in the intervention group, TIMEIN will be beneficial for enhancing adaptive ER strategies, reducing maladaptive ER strategies and lowering depressive symptoms. In contrast, it is expected that no such changes would occur in the control group. Furthermore, the second goal of the current study will be to tentatively explore whether TIME-IN is also accompanied by reductions in both parent - or

Social Science Protocols, July 2019, 1-16.

http://dx.doi.org/10.7565/ssp.2019.2651 
teacher reported EP and IP. To clear out whether any reductions in EP and IP are due to the impact of TIME-IN, we will control for natural fluctuations, related to children's regular development during this age period (see e.g. Bronfenbrenner, 1977) in a matched control group.

\section{Methods/Design}

\subsection{Study design}

The effectiveness of TIME-IN will be prospectively evaluated in a controlled pre-post study with two conditions: intervention (i.e. TIME-IN) versus matched controls selected from a group in regular education (see Figure 1). To test the effectiveness of TIME-IN, mechanisms and outcomes will be assessed through self-reported, parent-reported, and teacher-reported questionnaires before the start of the program (T0; pre-test at the beginning of the school year) and directly after finishing the program (T1; post-test at the end of the school year: 9 months later).

Figure 1. CONSORT diagram. Enrolment, T0 pre-test and T1 post-test.

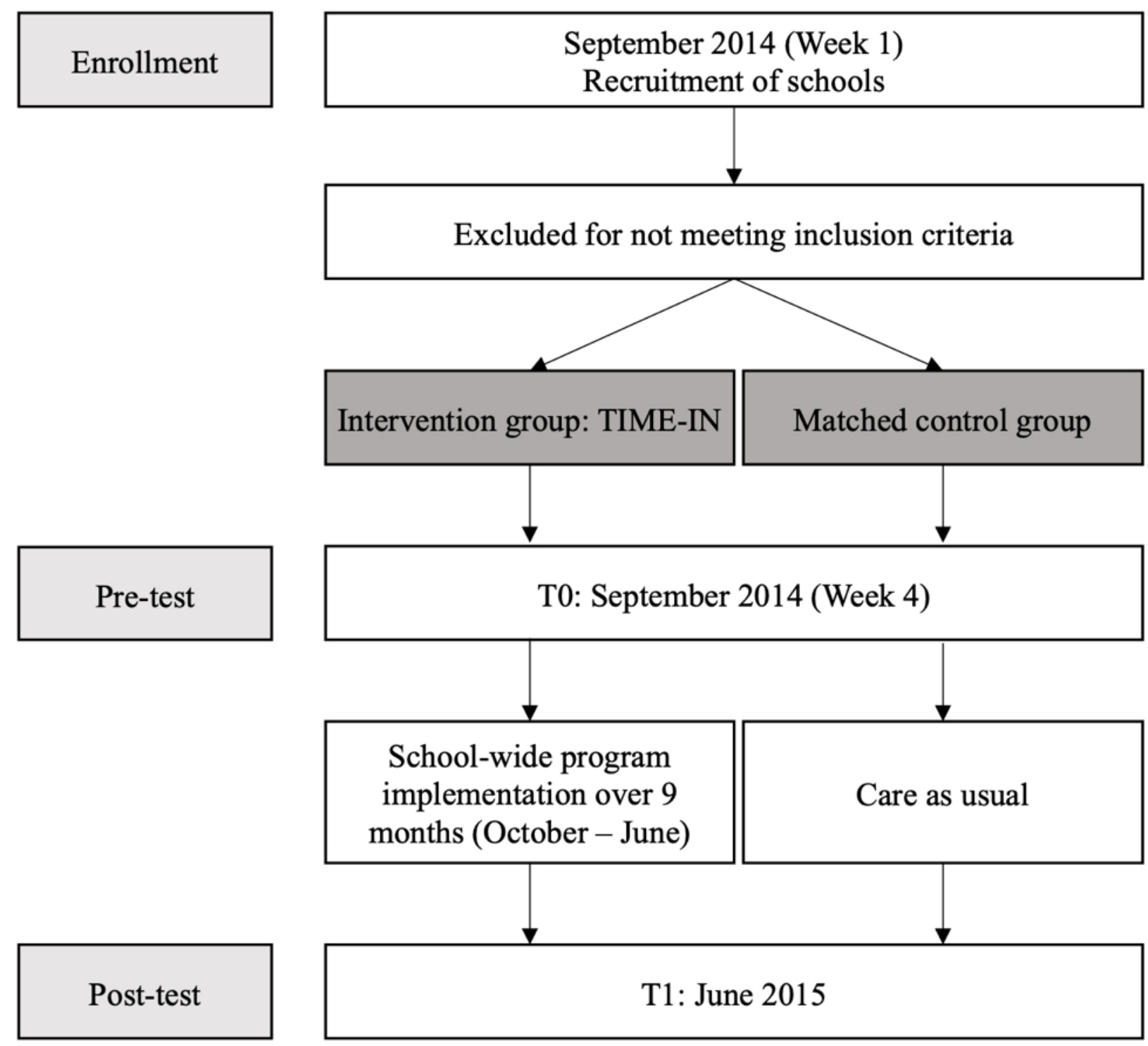

Social Science Protocols, July 2019, 1-16. 


\subsection{Participants}

One Flemish elementary school providing special education for children between 6 and 12 years of age with SEN was selected by the government as intervention group. Participants will be children between 8 and 12 years of age with SEN, as well as their teachers and parents. Since an opportunity sample will be included, the sample size will not be predetermined. Children, parents, and teachers who will not give their explicit consent to participate will be removed from the study. Furthermore, also all children that are too young to participate (i.e. 6-8 years old) or have a different SEN status (i.e. children with a severe mental health and/or physical disability) will be excluded from the study at the time of admission. Children for the control group, as well as their parents, will be randomly recruited from another ongoing study in 11 nearby elementary schools on school readiness (Generation 2020; Van Beveren, Mezulis, Wante, \& Braet, 2016) using SPSS case-control matching for age and gender. As Generation 2020 only focuses on screening children's school readiness and did not provide any interventions, the selected sample will be suitable to serve as control group. Finally, both the intervention and control group will be described in terms of age, gender, IQ, SES, and ethnic differences.

\subsection{Procedure}

A short presentation will be held in the intervention group to inform parents and teachers about the content of TIME-IN and the related research. Consequently, access will be provided to an online tool in order to be able to complete questionnaires at T0 and $\mathrm{T} 1$. Children will be asked to complete questionnaires on ER and depressive symptoms in both the intervention and the control group, while caregivers will be requested to complete questionnaires at home or in the classroom. More specifically, teachers will be asked to fill out a questionnaire on children's EP and IP in the intervention group, whereas parents will be requested to complete a comparable questionnaire in the control group. The overall data collection will be conducted by one additional researcher of Ghent University.

\subsection{TIME-IN: program content}

\section{Overall program structure}

TIME-IN has been published in a manual intended for supporting schools in developing a school-wide health care policy (Weymeis, 2015). This policy includes both behavioural (i.e. SWPBS) and emotional (i.e. screening IP, ART principles and LSCI) support systems, and this will be reflected in primary (all children), secondary (specific needs), and tertiary (individualized) practices (as defined by Sugai \& Horner, 2002). Both the schools' micro and meso level will be targeted as both levels need to be involved. Primary practices will be standardized regarding the number and duration of the sessions, whereas secondary and tertiary practices will be flexibly implemented and registered. An overview of all practices, as well as features of the program delivery, is presented in Figure 2. 
Figure 2. TIME-IN: Primary, Secondary, and Tertiary Practices.

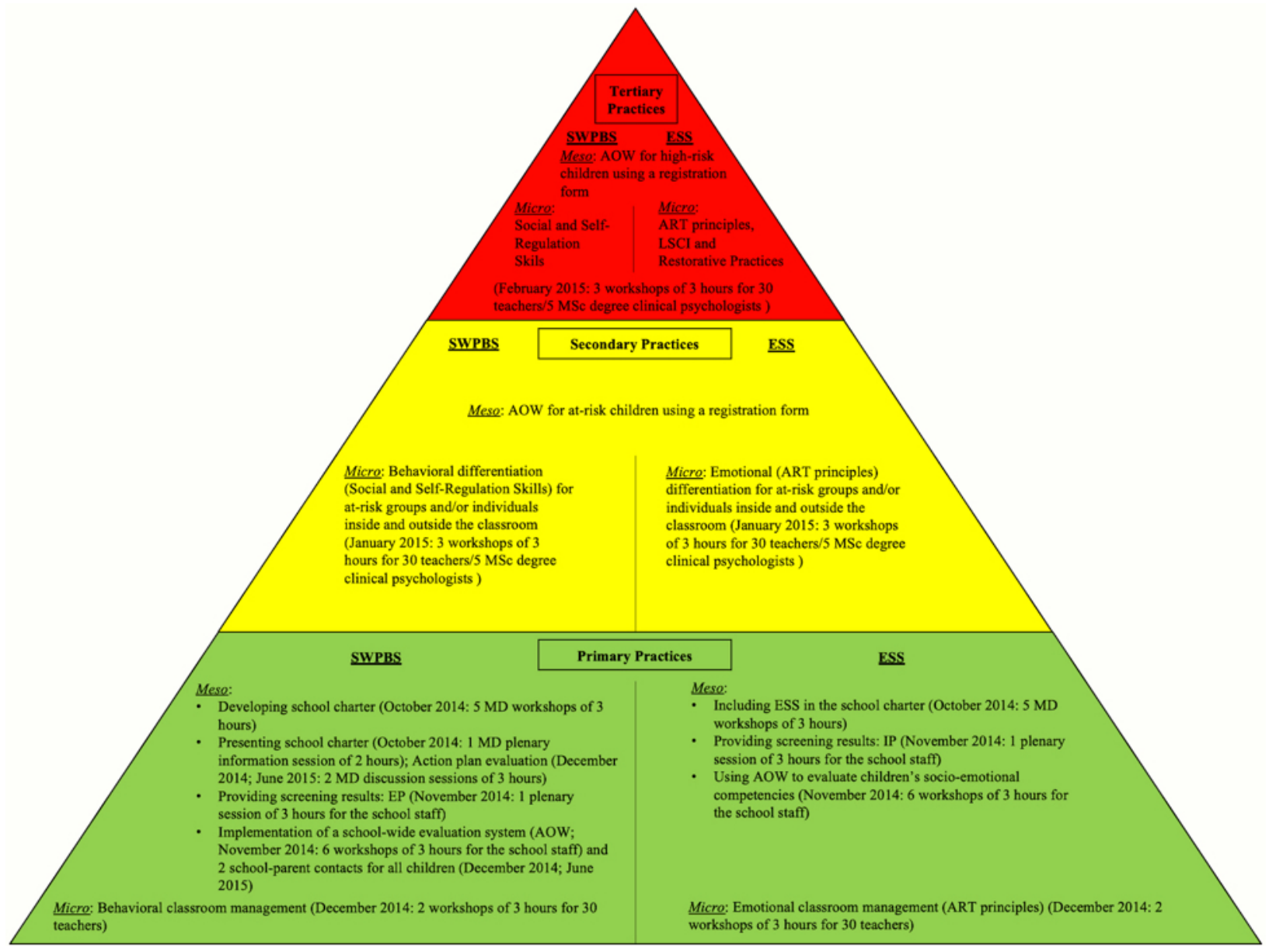

Note: SWPBS: School-Wide Positive Behaviour Support; ESS: Emotional Support Systems; $\mathrm{MD}=$ multidisciplinary; AOW $=$ Action-Oriented Working; ART $=$ Affect Regulation Training; LSCI: Life Space Crisis Intervention.

\subsection{School-Wide Positive Behaviour Support (SWPBS) interventions}

\section{a) Primary practices}

Within SWPBS, primary practices are intended to create a safe and predictable school and classroom environment (Sugai \& Horner, 2000) and include both meso and micro interventions. On the meso level, in order to prepare the school for organizational change, first, a school-wide charter will be developed and presented, with the objectives to raise attention for health care at school and to achieve consensus on whether and how health care will be organized. In doing so, both school staff, children, and their parents will be invited to cooperate as representing stakeholders by means of a school announcement and, consequently, the main agreements will be reflected in a written school-wide action plan (Sugai \& Horner, 2006). This action plan will be visually displayed within the school environment (e.g. the school hallway) and will be evaluated twice throughout the school year together with all representing stakeholders. Secondly, in addition to establishing a schoolwide charter, attention for the importance of health care at school will also be enhanced by raising teachers' awareness of children's overall degree of externalising problems. To do so, a reliable and valid screening instrument will be used to inform teachers about classroom average scores on EP (Achenbach \& Rescorla, 2001; Burke et al., 2012). Only if children Social Science Protocols, July 2019, 1-16.

http://dx.doi.org/10.7565/ssp.2019.2651 
and/or their parents give their explicit consent, caregivers will also be informed about children's individual subclinical or clinical scores for EP. Thirdly, analogous to the SWPBS evaluation instruments (Childs, Kincaid, \& George, 2010), a well-evaluated protocol for case formulation, action-oriented working (AOW; in Dutch: Handelingsgericht Werken; Pameijer, Van Beukering, De Lange, Schulpen, \& Van De Veire, 2010), will be implemented, in order to be able to efficiently and effectively translate the school's action plan into teachers' concrete classroom practice (Pameijer et al., 2010). On the one hand, AOW will be used to systematically evaluate all school-wide implemented practices, which will be supervised and tracked by one external instructor using monthly progress reports (Fixsen, Naoom, Blase, Friedman, \& Wallace, 2005). On the other hand, AOW will enable teachers and school psychologists to determine children's strengths and/or (special) educational needs (i.e. observing and understanding), as well as to select corresponding interventions, which will be evaluated in consultation with the children and their parents (i.e. planning and realizing). In doing so, all processes and outcomes will be catalogued using a registration form and, moreover, as research shows that parents' educational involvement is beneficial for promoting children's school readiness, two school-parent contacts will be structurally organized for all children throughout the school year (Seginer, 2006).

On the micro level, teachers will be expected to build on their behavioural classroom management for all children by (a) formulating and visualizing positive social and behavioural expectations (e.g. "if I need to get something, then I first ask the teacher"), (b) reinforcing children's positive behaviour, (c) providing clear instructions regarding the course content (e.g. "first, before starting, you will get a sheet with different pictures on it, which helps you to decide what you want to draw today"), and (d) using a visual stoplight to structurally introduce both behavioural expectations and class instructions (e.g. "red: teachers monitor behavioural expectations"; "orange: teachers monitor instructions" and "green: start of the lesson").

\section{b) Secondary and tertiary practices}

The project has a special classroom (i.e. a separate TIME-IN classroom) for organizing specific interventions for secondary (specific needs), and tertiary (individualized) practices. First, teachers and school psychologists will be trained in observing children's behaviours, as well as in conducting descriptive and functional analysis using the antecedent-behaviourconsequence model (Campbell \& Anderson, 2008; Ellis, 1985) to analyse each incident. Furthermore, children's social and self-regulation will be trained on both the subgroup and the individual level.

\subsection{Extending SWPBS with emotional support systems}

\section{a) Primary practices}

On the meso level, and as with SWPBS, first, emotional support systems will be included in the school's charter, as well as in the related school-wide action plan (Sugai \& Horner, 2006). Secondly, to raise teachers' awareness of children's psychosocial functioning, children's IP will be screened, as well as their emotional competencies. For the screening, reliable and valid screening instruments will be used to inform caregivers and teachers also about classroom average scores on IP (Achenbach \& Rescorla, 2001; McIntosh et al., 2014). Only if children and/or their parents give their explicit consent, caregivers will also be informed about children's individual subclinical or clinical IP scores.

Furthermore, on the micro level, teachers will also be expected to organize their emotional classroom management for all children. This is reflected in (a) a positive child-focused

Social Science Protocols, July 2019, 1-16.

http://dx.doi.org/10.7565/ssp.2019.2651 
attitude using verbal and non-verbal relational skills, (b) involving children's strengths and interests, (c) fuelling children's emotional awareness by using a classroom emotional thermometer tool, (d) enhancing emotion identification by visualizing different emotions, and (e) fostering emotional understanding (i.e. linking emotions to situations, thoughts, and behaviours) by using the ABC - model (Bergin \& Bergin, 2009; Berking \& Schwarz, 2014; Ellis, 1985).

\section{b) Secondary and tertiary practices}

On the micro level, secondary practices will consist of coaching adaptive ER strategies (e.g. problem solving, acceptance and cognitive reappraisal) in the classroom by means of a reminder card showing a specific instruction, every time the teacher sees an opportunity. In some cases, this will be complemented by a more extensive ER training provided by a school psychologist outside of the classroom, tailored to children's special educational needs. Furthermore, on the tertiary level, when aggressive behaviour or an emotional crisis is likely to arise, individualized practices consist of the Life Space Crisis Intervention procedure (Long et al., 2003), as well as restorative practices that will focus on repairing conflictual relationships among children and/or between teacher(s) and child(ren) (McCluskey et al., 2008). During the roll-out of these procedures, teachers will be able to support children in cooling down within the classroom or, in the case of a crisis, to refer children to a school psychologist for further assistance outside the classroom.

\section{c) Program delivery}

The implementation of interventions is spread over 9 months and consists of 60 meetings. Primary practices are implemented from October 2014, secondary practices from January 2015, and tertiary practices from February 2015. More specifically, on the meso level, 5 multidisciplinary (MD; i.e. one external instructor, school staff, and parents) workshops of 3 hours and $1 \mathrm{MD}$ plenary information session of 2 hours will be devoted to the development and presentation of a school-wide charter, while 2 MD discussion sessions of 3 hours will be planned throughout the school year to evaluate the schools' overall action plan. Next, 1 information session of 3 hours will be organized for the school's staff regarding screening results for EP and IP, as well as 6 workshops of 3 hours for the implementation of AOW (Pameijer et al., 2010).

Furthermore, on the micro level, to be able to accurately apply both primary, secondary, and tertiary practices within the classroom, 30 teachers will be trained during 13 workshops of 3 hours. Also, the school board, which consists of $5 \mathrm{MSc}$ degree clinical psychologists or school pedagogues, will receive 9 workshops of 3 hours for training in outside classroom interventions. Finally, 2 school-parent contacts of 3 hours will be proactively organized to involve all parents in promoting the children's learning process.

All workshops will involve small interactive groups of up to 15 participants, to whom information and examples will be presented. Also, each group will have the opportunity to practice the required skills by using specific tools (Fixsen et al., 2005). Moreover, as training sessions seem to be only efficient when paired with consulting, 21 intermediate MD consultation meetings (i.e. one external instructor, school staff, and if possible, also the parents) of 2 hours will be organized for further supervision, supporting teachers and parents throughout the school year. During these meetings, teachers and parents will be given the opportunity to discuss their personal support needs, as well as children's SEN. 


\subsection{Assessments and measures}

\section{a) Primary outcomes}

Adaptive and maladaptive emotion regulation strategies: FEEL-KJ. To measure children's use of ER strategies, the Fragebogen zur Erhebung der Emotionsregulation bei Kindern und Jugendlichen will be used (FEEL-KJ; Grob \& Smolenski, 2005); Dutch version by Braet, Cracco, and Theuwis (2013). The FEEL-KJ is a 90-item child-report questionnaire for measuring a broad range of ER strategies in children and adolescents between 8 and 18 years old. Each strategy is measured by rating two items for each of the three emotions, whereby answers are given on a 5-point Likert scale $(1=$ almost never; $2=$ rarely; 3 =occasionally; 4 $=$ often; 5 = almost always). Regarding psychometric qualities, the FEEL-KJ is wellvalidated and reliable (Cracco, Van Durme, \& Braet, 2015).

Depressive symptoms: CDI. The Children's Depression Inventory (CDI; Kovacs, 1992); Dutch version by Timbremont and Braet (2002) is a 27-item self-report questionnaire for assessing cognitive, affective, and behavioural symptoms of depression in children and adolescents between 7 and 17 years of age. Answers for each item are given on a 3-point Likert scale indicating level of severity. Regarding psychometric qualities, the CDI is shown to be well-validated and reliable (Craighead, Smucker, Craighead, \& Ilardi, 1998; Smucker, Craighead, Craighead, \& Green, 1986).

\section{b) Secondary outcomes}

Psychosocial problems: TRF and CBCL. The Teacher Report Form (intervention group) and Child Behaviour Checklist (control group) (Achenbach \& Rescorla, 2001); Dutch version by Verhulst, Van der Ende, and Koot (1996; 1997) respectively, are 113-item teacher-report questionnaires for measuring teachers' and parents' perceptions of 6 to 18-year-old children's adaptive and maladaptive functioning. Regarding psychometric qualities, the TRF and CBCL are well-validated and reliable (Achenbach, Dumenci, \& Rescorla, 2003). In the intervention study, children's teachers reported on EP and IP, whereas in the control group, parents were informants of children's EP and IP. Comparing parents' and teachers' reports on these measures seems reasonable, as previous research showed modest cross-informant agreement between parents and teachers regarding children's EP and IP (Achenbach, Dumenci, \& Rescorla, 2002). However, this is only for descriptive purposes as we are primarily interested in the (experimentally manipulated or naturally) fluctuations between the scores during the 9month project thereby comparing pre-test versus post-test scores of the same informant.

\subsection{Data analytic plan}

Firstly, Little's MCAR test will be performed to determine the rate of missing data and, furthermore, to determine whether data is missing completely at random (Little, 1988). If so, missing data will be estimated using multiple imputations. Secondly, descriptive statistics, correlations, and the distribution of children in the normal, subclinical or clinical range will be calculated and presented. Also, the assumption of normality will be tested. Thirdly, twotailed independent $t$-tests will be performed to check whether the means of the study variables differ significantly between the intervention and the control group at baseline. Cohen's (1992) effect size $(E S) d$ will be calculated to determine the size of mean differences.

Fourthly and finally, the main study hypotheses will be examined by performing separate two-way repeated measures ANCOVA's for each outcome variable (i.e. time x condition). Regarding the first study hypothesis, adaptive ER will be controlled for levels of maladaptive ER, while maladaptive ER will be controlled for levels of adaptive ER, because adaptive ER and maladaptive ER seem to be correlated (Cracco et al., 2015). Regarding the second study Social Science Protocols, July 2019, 1-16.

http://dx.doi.org/10.7565/ssp.2019.2651 
hypothesis, gender differences will be controlled for, as it is known from the literature that girls typically experience more IP compared to boys (Crijnen et al., 1997). As EP and IP commonly interfere with each other, EP will be controlled for levels of IP, while IP will be controlled for levels of EP (Masten et al., 2005). Cohen's effect size $f$ will be calculated to determine the interventions' impact magnitude and the level of significance for all analyses will be set at $p<.05$. Fourthly and finally, to determine whether there is a clinically significant change in the intervention group for both the primary and the secondary outcome variables, the Reliable Change Index (RCI) will be calculated using the formula from Jacobson and Truax (1991). When the RCI is higher than 1.96, the post-test score is likely to reflect a real change.

\section{Expected Results}

The anticipated results (see Figure 3) were based on norms for the study variables that (a) were reported in the manual, and (b) take into account age and gender (see Table 1; Braet, Cracco, \& Theuwis, 2013; Timbremont \& Braet, 2002; Verhulst, Van der Ende, \& Koot, 1997). Generally, it was expected that the proposed hypotheses will be confirmed after implementing the TIME-IN protocol. More specifically, in the intervention group, children with special educational needs will be involved, from whom is expected that some but not all cases will exhibit subclinical psychosocial difficulties (i.e. at-risk group; statistically $1 S D$ from the mean). Therefore, it is reasonable to assume (a) averaged mean post-test scores or (b) mean post-test scores in the average range. In contrast, the control group will mainly include children without special educational needs, from whom it is expected that only a small minority faces psychosocial problems. Therefore, and since no interventions will be provided in this group, it is fair to believe that non-clinical mean baseline values will remain stable during the course of the school year.

Table 1. Norms for the Study Variables and Expected Results.

\begin{tabular}{|c|c|c|c|c|c|c|}
\hline \multirow{3}{*}{$\begin{array}{l}\text { Study } \\
\text { variables }\end{array}$} & \multirow{3}{*}{$\begin{array}{l}\text { Norm } \\
\text { group }\end{array}$} & \multirow{3}{*}{$\begin{array}{l}\text { Age } \\
\text { category }\end{array}$} & \multicolumn{4}{|c|}{ TIME-IN } \\
\hline & & & \multicolumn{2}{|c|}{ (a) Averaged scores } & \multicolumn{2}{|c|}{ (b) Average range } \\
\hline & & & Boys & Girls & Boys & Girls \\
\hline Adaptive ER & Flemish & $8.0-12.0$ & $104.8+28.9$ & $111.2+27.2$ & $133.8 \pm 28.9$ & $138.4 \pm 27.2$ \\
\hline $\begin{array}{l}\text { Maladaptive } \\
\text { ER }\end{array}$ & Flemish & $8.0-12.0$ & $87.4-15.6$ & $89.8-14.6$ & $71.8 \pm 15.6$ & $75.2 \pm 14.6$ \\
\hline $\begin{array}{l}\text { Depressive } \\
\text { symptoms }\end{array}$ & Flemish & $8.0-12.0$ & $14.3-5.9$ & $15.4-6.5$ & $8.4 \pm 5.9$ & $8.9 \pm 6.5$ \\
\hline $\begin{array}{l}\text { Externalising } \\
\text { problems }\end{array}$ & Dutch & $4.0-11.0$ & $15.7-9.0$ & $8.4-5.2$ & $6.7 \pm 9.0$ & $3.2 \pm 5.1$ \\
\hline $\begin{array}{l}\text { Internalising } \\
\text { problems }\end{array}$ & Dutch & $4.0-11.0$ & $11.8-6.3$ & $11.3-6.1$ & $5.6 \pm 6.3$ & $5.2 \pm 6.1$ \\
\hline
\end{tabular}

Note: Data were presented as (a) subclinical value + or $-1 S D$, or (b) mean $\pm 1 S D$. ER $=$ emotion regulation. 
Figure 3. TIME-IN: Expected results.
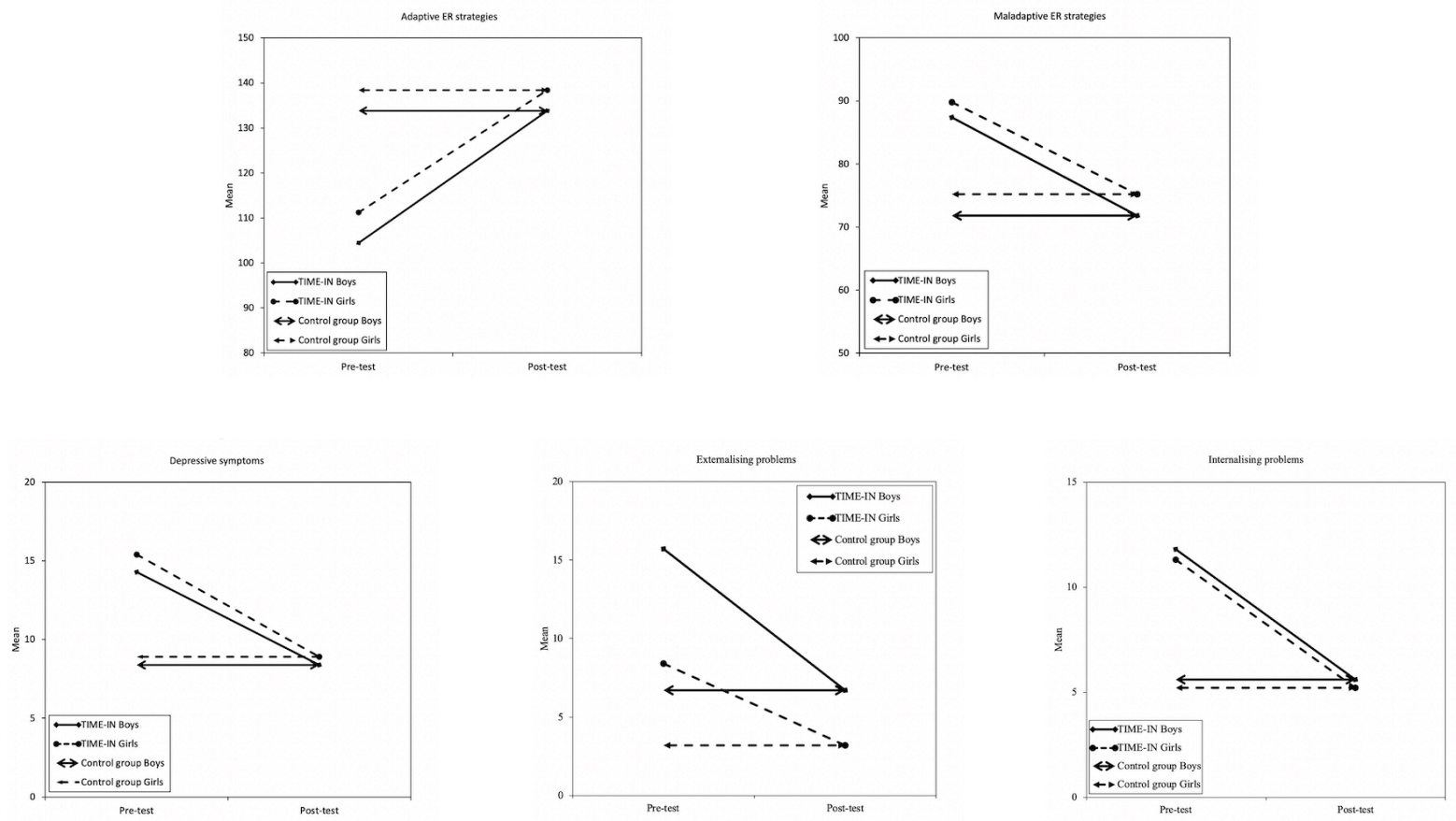

\section{Discussion}

The current study will evaluate the effectiveness of TIME-IN, a school-wide health care policy for promoting school readiness in children with SEN. TIME-IN aims to extend SWPBS and its behavioural interventions by providing emotional support systems, which are intended for strengthening children's emotional competencies, as well as for reducing both EP and IP. More specifically, in a controlled pre-post study consisting of an intervention and matched control group, first, the confirmatory hypotheses will be tested whether TIME-IN would be beneficial for improving children's use of adaptive ER strategies, reducing maladaptive ER strategies and lowering depressive symptoms. Secondly, the exploratory hypotheses will be tested whether a reduction of EP and IP would occur in the intervention group and whether the same (natural) fluctuations were observable in a control group.

\section{Practical Implications}

If TIME-IN significantly improves children's school readiness, this entails different practical implications for schools and teachers in educating children with SEN. More specifically, first, schools and teachers are often convinced that disciplinary practices are the most effective way to address children's behavioural and emotional problems. In this regard, a beneficial impact of TIME-IN on children's school readiness can deliver a rationale to facilitate the implementation of a positively approached school-wide health care policy (Beets et al., 2008; Forman, Olin, Hoagwood, Crowe, \& Saka, 2009; Sugai \& Horner, 2002). In addition, as implementation efforts are often accompanied by stress, feelings of incompetence, and resistance to change, promising study results may convince teachers to participate during sustained implementation efforts (Evers, Brouwers, \& Tomic, 2002). Secondly, the results may suggest that, besides the implementation of well-known behavioural practices, it is useful for schools to also include emotional support systems in special education (McIntosh et al., 2014). More specifically, to enable children to deal with

Social Science Protocols, July 2019, 1-16. 
academic stress and related emotions, teachers could be trained in screening and training children's emotional competencies such as adaptive ER strategies (Ben-Eliyahu \& Linnenbrink-Garcia, 2013; Davis \& Levine, 2012). Thirdly and finally, the results may yield useful insights into how children with SEN could be better (re)integrated into regular education. If children are able to efficiently manage their emotions, they might also better withstand challenging school demands (Graziano, Reavis, Keane, \& Calkins, 2007).

\section{Strengths and Limitations}

Finally, both study limitations and strengths will be considered. Regarding study strengths, first, reliable measures will be used, which decreases possible error variance and, as such, increases the study's statistical power. Secondly, the current study has the potential to compensate for shortcomings in experimental research as it will be conducted in a real-life setting and, as a result, will provide 'richer' data and increased ecological validity (Schmuckler, 2001). Thirdly, the current study includes longitudinal data, as well as a matched control group, which may yield preliminary signs of causality on the assumed relations between the study variables (Maxwell \& Cole, 2007; Simons et al., 2016; Veerman $\&$ Van Yperen, 2007). This implicates that, within a short time range of 9 months, modest statements can be made about the effects of TIME-IN on children's EP and IP, as well as on their use of ER strategies. Fourthly and finally, this will be one of the first studies investigating a school-wide intervention that aims to extend SWPBS by adding emotional support systems and, moreover, by specifically focusing on improving children's emotional readiness.

Regarding study limitations, first, as we include only one school for the intervention, the sample included could be too small for strong statistical power and, moreover, this reduces the chance of obtaining reliable and generalizable results (see also Parker, 1990). Therefore, for the current sample, post hoc power analysis will be performed to find out which sample size is appropriate to generate sufficient power (i.e. 1- $\beta$ ). Secondly, regarding the study design, there is a lack of randomization, which increases the chance that uncontrolled factors will be unevenly distributed over the intervention and the control group. Moreover, although both conditions will be matched regarding demographical characteristics such as gender and age, baseline scores for the intervention and control group can significantly differ, which might suggest that we are not able to take into account the influence of other both child (e.g. SEN or not) or environmental (e.g. educational context) factors. Both problems reduce the ability to draw causal conclusions about the effect of TIME-IN on children's school readiness (Simons et al., 2016). To resolve these issues, and to be able to conclude that TIME-IN is efficacious, evidence is required that the presumed outcomes are caused by the intervention and/or its presumed working mechanisms (Veerman \& van Yperen, 2007). In this regard, causal statements typically arise from rigorous evaluations such as a randomized controlled trial (RCT) and/or a single case study. For the moment, we have not yet been able to carry out such research. Thirdly, another study limitation is related to the single use of questionnaires, which could result in shared method variance. This issue could, however, be addressed in the future by including other data sources such as observations, interviews, and children's concrete test results. Fourthly and finally, some issues may occur due to the use of teacher and/or parent reports for our secondary outcome measures. Scores for EP and IP were obtained by different informants in the intervention and control group, which could lead to distorted or tentative results (Simons et al., 2016), as it complicates a reliable comparison. As we are interested in the (experimentally manipulated or naturally) fluctuations between the scores during the 9-month project thereby comparing pre-test versus post-test scores of the same informant, we believe the findings on IP and EP could be informative to include.

Social Science Protocols, July 2019, 1-16.

http://dx.doi.org/10.7565/ssp.2019.2651 


\section{Conclusion}

The current intervention study will investigate whether TIME-IN, which extends SWPBS by adding emotional support systems, is beneficial for fostering children's school readiness. If so, the results may provide indicative evidence that TIME-IN improves children's psychosocial functioning, which, as a result, may convince schools and teachers to also sustainably implement emotional practices as a classroom management strategy (Beets et al., 2008; Veerman \& van Yperen, 2007).

\section{Declarations}

Competing interests: Braet, Cracco, \& Theuwis (2013) translated the FEEL-KJ (Grob \& Smolenski, 2005) and received an authorship fee for the official version.

\section{References}

Abidin, R. R., \& Robinson, L. L. (2002). Stress, biases, or professionalism: what drives teachers' referral judgments of students with challenging behaviours? Journal of Emotional and Behavioural Disorders, 10(4), 204-212. doi:10.1177/10634266020100040201

Achenbach, T., Dumenci, L., \& Rescorla, L. (2002). Ten-year comparisons of problems and competencies for national samples of youth: self, parent, and teacher reports. 10, 194-203.

Achenbach, T. M., Dumenci, L., \& Rescorla, L. A. (2003). DSM-oriented and empirically based approaches to constructing scales from the same item pools. Journal of Clinical Child and Adolescent Psychology, 32(3), 328-340. doi:10.1207/S15374424jccp3203_02

Achenbach, T. M., \& Rescorla, L. (2001). Manual for the ASEBA school-age forms \& profiles: an intergrated system of mult-informant assessment. Brulington: VT: University of Vermont, Research Center for Children, Youth, \& Families.

Aldao, A., Gee, D. G., De Los Reyes, A., \& Seager, I. (2016). Emotion regulation as a transdiagnostic factor in the development of internalising and externalising psychopathology: Current and future directions. Development and Psychopathology, 28(4), 927-946. doi:10.1017/S0954579416000638

Aldao, A., Nolen-Hoeksema, S., \& Schweizer, S. (2010). Emotion-regulation strategies across psychopathology: A meta-analytic review. Clinical Psychology Review, 30(2), $217-$ 237. doi:10.1016/j.cpr.2009.11.004

Baker, J. A., Grant, S., \& Morlock, L. (2008). The teacher-student relationship as a developmental context for children with internalising or externalising behaviour problems. School Psychology Quarterly, 23(1), 3-15. doi:10.1037/1045-3830.23.1.3

Beets, M., Flay, B., Vuchinich, S., Acock, A., Li, K. K., \& Allred, C. (2008). School climate and teachers' beliefs and attitudes associated with implementation of the positive action program: a diffusion of innovations model. Prevention Science, 9(4), 264-275. doi:10.1007/s11121-008-0100-2

Ben-Eliyahu, A., \& Linnenbrink-Garcia, L. (2013). Extending self-regulated learning to include self-regulated emotion strategies. Motivation and Emotion, 37(3), 558-573. doi:10.1007/s11031-012-9332-3

Bergin, C., \& Bergin, D. (2009). Attachment in the Classroom. Educational Psychology Review, 21(2), 141-170. doi:10.1007/s10648-009-9104-0

Berking, M., \& Lukas, C. A. (2015). The Affect Regulation Training (ART): a transdiagnostic approach to the prevention and treatment of mental disorders. Current Opinion in Psychology, 3, 64-69. doi:10.1016/j.copsyc.2015.02.002

Social Science Protocols, July 2019, 1-16.

http://dx.doi.org/10.7565/ssp.2019.2651 
Berking, M., \& Schwarz, J. (2014). Affect Regulation Training. In J. J. Gross (Ed.), Handbook of emotion regulation (pp. 1-19).

Braet, C., Cracco, E., \& Theuwis, L. (2013). Questionnaire for emotion regulation in children and adolescents. Amsterdam: Hogrefe.

Britto, P. R. (2012). School readiness: A conceptual framework. Retrieved from New York: https://www.unicef.org/education/files/Chil2Child_ConceptualFramework_FINAL(1).pdf

Bronfenbrenner, U. (1977). Toward an experimental ecology of human-development. American Psychologist, 32(7), 513-531. doi:10.1037/0003-066x.32.7.513

Burke, M. D., Davis, J. L., Lee, Y. H., Hagan-Burke, S., Kwok, O. M., \& Sugai, G. (2012). Universal screening for behavioural risk in elementary schools using swpbs expectations. Journal of Emotional and Behavioural Disorders, 20(1), 38-54. doi:10.1177/1063426610377328

Campbell, A., \& Anderson, C. M. (2008). Enhancing effects of check-in/check-out with function-based support. Behavioural Disorders, 33(4), 233-245.

Childs, K. E., Kincaid, D., \& George, H. P. (2010). A model for statewide evaluation of a universal positive behaviour support initiative. Journal of Positive Behaviour Interventions, 12(4), 198-210. doi:10.1177/1098300709340699

Cohen, J. (1992). A power primer. Psychological Bulletin, 112(1), 155-159. doi:10.1037/0033-2909.112.1.155

Cracco, E., Van Durme, K., \& Braet, C. (2015). Validation of the FEEL-KJ: an instrument to measure emotion regulation strategies in children and adolescents (vol 10, e0137080, 2015). Plos One, 10(12). doi:10.1371/journal.pone.0144324

Craighead, W. E., Smucker, M. R., Craighead, L. W., \& Ilardi, S. S. (1998). Factor analysis of the Children's Depression Inventory in a community sample. Psychological Assessment, 10(2), 156-165. doi:10.1037//1040-3590.10.2.156

Crijnen, A. A. M., Achenbach, T. M., \& Verhulst, F. C. (1997). Comparisons of problems reported by parents of children in 12 cultures: total problems, externalising, and internalising. Journal of the American Academy of Child and Adolescent Psychiatry, 36(9), 1269-1277. doi:10.1097/00004583-199709000-00020

Davis, E. L., \& Levine, L. J. (2012). Emotion regulation strategies that promote learning: reappraisal enhances children's memory for educational information. Child Development, 84(1), 361-374. doi:10.1111/j.1467-8624.2012.01836.x

Dawson, C. A. (2003). A study on the effectiveness of life space crisis intervention for students identified with emotional disturbances. Reclaiming Children and Youth, 11(4), 223-230. Retrieved from https://search.proquest.com/docview/214201649?accountid=11077

Dekker, M. C., Koot, H. M., van der Ende, J., \& Verhulst, F. C. (2002). Emotional and behavioural problems in children and adolescents with and without intellectual disability. Journal of Child Psychology and Psychiatry and Allied Disciplines, 43(8), 1087-1098. doi:10.1111/1469-7610.00235

Ellis, A. (1985). Expanding the ABCs of RET. In M. J. Mahoney \& F. A. (Eds.), Cognition and psychotherapy (pp. 313-323). New York: Plenum.

Evers, W. J. G., Brouwers, A., \& Tomic, W. (2002). Burnout and self-efficacy: a study on teachers' beliefs when implementing an innovative educational system in the Netherlands. British Journal of Educational Psychology, 72, 227-243. doi:10.1348/000709902158865

Fixsen, D. L., Naoom, S. F., Blase, A. B., Friedman, R. M., \& Wallace, F. (2005). Implementation research: a synthesis of the literature. Tampa, FL: University of South Florida, Louis de la Parte Florida Mental Health Institute: The National Implementation Research Network (FMHI Publication \#231). 
Forman, S. G., Olin, S. S., Hoagwood, K. E., Crowe, M., \& Saka, N. (2009). Evidence-Based interventions in schools: developers' views of implementation barriers and facilitators. School Mental Health, 1, 26-36. doi:10.1007/s12310-008-9002-5

Graziano, P. A., Reavis, R. D., Keane, S. P., \& Calkins, S. D. (2007). The role of emotion regulation in children's early academic success. Journal of School Psychology, 45(1), 319. doi:10.1016/j.jsp.2006.09.002

Grob, A., \& Smolenski, C. (2005). Fragebogen zur Erhebung der Emotionsregulation bei Kindern und Jugendlichen. Bern: Huber.

Gross, J. J. (1998). Antecedent- and response-focused emotion regulation: divergent consequences for experience, expression, and physiology. Journal of Personality and Social Psychology, 74(1), 224-237. doi:10.1037//0022-3514.74.1.224

Horner, R. H., \& Sugai, G. (2000). School-Wide Behaviour Support: An Emerging Initiative. Journal of Positive Behaviour Interventions, 2(4), 231-232. doi:10.1177/109830070000200407

Horner, R. H., Sugai, G., \& Anderson, C. M. (2010). Examining the evidence base for School-Wide Positive Behaviour Support. Focus on Exceptional Children, 42(8), 1-14.

Horner, R. H., Sugai, G., Smolkowski, K., Eber, L., Nakasato, J., Todd, A. W., \& Esperanza, J. (2009). A randomized, wait-list controlled effectiveness trial assessing School-Wide Positive Behaviour Support in elementary schools. Journal of Positive Behaviour Interventions, 11(3), 133-144. doi:10.1177/1098300709332067

Jacobson, N.S. and Truax, P. (1991). Clinical significance: a statistical approach to defining meaningful change in psychotherapy research. Journal of Consulting and Clinical Psychology. 59(1), 12-19. doi:10.1037/0022-006X.59.1.12

Keil, V., \& Price, J. M. (2006). Externalising behaviour disorders in child welfare settings: Definition, prevalence, and implications for assessment and treatment. Children and Youth Services Review, 28(7), 761-779. doi:10.1016/j.childyouth.2005.08.006

Kovacs, M. (1992). Children's Depression Inventory. New York: Multi-Health Systems.

Little, R. J. A. (1988). A test of missing completely at random for multivariate data with missing values. Journal of the American Statistical Association, 83(404), 1198-1202. doi: $10.2307 / 2290157$

Long, N. J., Wood, M. M., \& Fecser, F. A. (2003). Life Space Crisis Intervention. Tielt: Lannoo Campus.

Masten, A. S., Roisman, G. I., Long, J. D., Burt, K. B., Obradovic, J., Riley, J. R., . . . Tellegen, A. (2005). Developmental cascades: linking academic achievement and externalising and internalising symptoms over 20 years. Developmental Psychology, 41(5), 733-746. doi:10.1037/0012-1649.41.5.733

Maxwell, S. E., \& Cole, D. A. (2007). Bias in cross-sectional analyses of longitudinal mediation. Psychological Methods, 12(1), 23-44. doi:10.1037/1082-989x.12.1.23

McCluskey, G., Lloyd, G., Kane, J., Riddell, S., Stead, J., \& Weedon, E. (2008). Can restorative practices in schools make a difference? Educational Review, 60(4), 405-417. doi:10.1080/00131910802393456

McIntosh, K., Ty, S. V., \& Miller, L. D. (2014). Effects of School-Wide Positive Behavioural Interventions and Supports on internalising problems: current evidence and future directions. Journal of Positive Behaviour Interventions, 16(4), 209-218. doi:10.1177/1098300713491980

Morgan, P. L., Farkas, G., Tufis, P. A., \& Sperling, R. A. (2008). Are reading and behaviour problems risk factors for each other? Journal of Learning Disabilities, 41(5), 417-436. doi:10.1177/0022219408321123 
Murray, C., \& Murray, K. M. (2004). Child level correlates of teacher-student relationships: An examination of demographic characteristics, academic orientations, and behavioural orientations. Psychology in the Schools, 41(7), 751-762. doi:10.1002/pits.20015

Ollendick, T. H., \& King, N. J. (1994). Diagnosis, assessment, and treatment of internalising problems in children - the role of longitudinal data. Journal of Consulting and Clinical Psychology, 62(5), 918-927. doi:10.1037/0022-006x.62.5.918

Pameijer, N., Van Beukering, T., De Lange, S., Schulpen, Y., \& Van De Veire, H. (2010). Handelingsgericht werken in de klas. Leuven: Acco.

Parker, R. M. (1990). Power, Control, and Validity in Research. Journal of Learning Disabilities, 23(10), 613-620. doi:10.1177/002221949002301008

Schmuckler, M. A. (2001). What is ecological validity? a dimensional analysis. Infancy, 2(4), 419-436. doi:10.1207/S15327078in0204_02

Seginer, R. (2006). Parents' educational involvement: A developmental ecology perspective. Parenting-Science and Practice, 6(1), 1-48. doi:10.1207/s15327922par0601_1

Shaw, D. S., Keenan, K., Vondra, J. I., Delliquadri, E., \& Giovannelli, J. (1997). Antecedents of preschool children's internalising problems: A longitudinal study of low-income families. Journal of the American Academy of Child and Adolescent Psychiatry, 36(12), 1760-1767. doi:10.1097/00004583-199712000-00025

Simons, D. J., Boot, W. R., Charness, N., Gathercole, S. E., Chabris, C. F., Hambrick, D. Z., \& Stine-Morrow, E. A. L. (2016). Do "Brain-Training" programs work? Psychological Science in the Public Interest, 17(3), 103-186. doi:10.1177/1529100616661983

Smucker, M. R., Craighead, W. E., Craighead, L. W., \& Green, B. J. (1986). Normative and reliability data for the Childrens Depression Inventory. Journal of Abnormal Child Psychology, 14(1), 25-39. doi:10.1007/Bf00917219

Soenen, B., Volckaert, A., D’Oosterlinck, F., \& Broekaert, E. (2014). The implementation of Life Space Crisis Intervention in residential care and special education for children and adolescents with EBD: An Effect Study. Psychiatric Quarterly, 85(3), 267-284. doi:10.1007/s11126-014-9288-4

Sugai, G., \& Horner, R. (2002). The evolution of discipline practices: School-wide positive behaviour supports. Child \& Family Behaviour Therapy, 24(1-2), 23-50. doi:10.1300/J019v24n01_03

Sugai, G., Horner, R., Dunlap, G., Hieneman, M., Lewis, T. J., Nelso, C. M., . . Turnbull, H. R. (2000). Applying Positive Behaviour Support and functional behavioural assessment in schools. Journal of Positive Behaviour Interventions, 2(3), 131-143. doi:10.1177/109830070000200302

Sugai, G., \& Horner, R. R. (2006). A promising approach for expanding and sustaining school-wide positive Behaviour support. School Psychology Review, 35(2), 245-259.

Timbremont, B., \& Braet, C. (2002). Children's Depression Inventory: Nederlandstalige versie [Children's Depression Inventory: Dutch version]. Lisse: Swets \& Zeitlinger.

Van Beveren, M. L., Mezulis, A., Wante, L., \& Braet, C. (2016). Joint contributions of negative emotionality, positive emotionality, and effortful control on depressive symptoms in youth. Journal of Clinical Child \& Adolescent Psychology, 2, 1-12. doi:10.1080/15374416.2016.1233499

Van der Ende, J., Verhulst, F. C., \& Tiemeier, H. (2016). The bidirectional pathways between internalising and externalising problems and academic performance from 6 to 18 years. Development and Psychopathology, 28(3), 855-867. doi:10.1017/S0954579416000353

Veerman, J. W., \& van Yperen, T. A. (2007). Degrees of freedom and degrees of certainty: a developmental model for the establishment of evidence-based youth care. Evaluation and Program Planning, 30(2), 212-221. doi:10.1016/j.evalprogplan.2007.01.011 
Verhulst, F. C., Van der Ende, J., \& Koot, H. M. (1996). Manual for the CBCL/4-18.

Rotterdam: Afdeling Kinder-en Jeugdpsychiatrie, Sophia. Kinderziekenhuis/Academisch Ziekenhuis Rotterdam, Erasmus Universiteit Rotterdam.

Verhulst, F. C., Van der Ende, J., \& Koot, H. M. (1997). Dutch Manual for the Teacher's Report Form (TRF). Rotterdam: Afdeling Kinder-en Jeugdpsychiatrie, Sophia. Kinderziekenhuis/Academisch Ziekenhuis Rotterdam, Erasmus Universiteit Rotterdam.

Westwood, P. (2007). Commonsense methods for children with special educational needs (5 ed.). London, UK: Routledge.

Weymeis, H. (2015). Wij Zijn Gedrag: Positief Gedrag In Het Onderwijs. Leuven, Belgium: Acco.

Wolff, J. C., \& Ollendick, T. H. (2006). The comorbidity of conduct problems and depression in childhood and adolescence. Clinical Child and Family Psychology Review, 9(3-4), 201220. doi:10.1007/s10567-006-0011-3

World Health Organization. (2012). International Standards for Clinical Trial Registries. Geneva, Switzerland: World Health Organization Retrieved from http://apps.who.int/iris/bitstream/10665/76705/1/9789241504294_eng.pdf?ua=1.

Yoon, J. S. (2002). Teacher characteristics as predictors of teacher-student relationships: Stress, negative affect, and self-efficacy. Social Behaviour and Personality, 30(5), 485 493. doi:10.2224/sbp.2002.30.5.485 